\title{
Image Compression by a Time Enhanced Self Organizing Map
}

\author{
Pascual Campoy and Pedro Gutiérrez* \\ Departamento de Automática, Ingeniería Electrónica e Informática Industrial \\ Universidad Politécnica de Madrid, Spain \\ pascual.campoy@upm.es, pgutierrez@etsii.upm.es
}

\begin{abstract}
This paper presents the promising results of an innovative modification of the Kohonen's algorithm, the time enhanced self-organizing map (TESOM), when used for low bitrate image compression. The time enhanced map is used in this paper to learn codebooks of subimages, in a similar way as other classical algorithms based on LVQ or SOM do, but taking advantage of the fact that it learns the sequence order of the input data (i.e. subimages) during the training phase. The codebook learned by the new proposed algorithm TESOM presents the advantage that the vicinity of the codes in the output map is not only established by their visual similarity, as in SOM, but also by the sequential order of the subimages during the training phase. Since this sequential order of the subimages determines the vicinity of the codes, the increment of the representative code of two consecutive subimages has been proved to have a lover Entropy and can therefore be codified by a lower bit rate. The advantage of the proposed algorithm is thoroughly evaluated and quantified over a set of experiments, which include several images, used in different ways in the training phase for codebook design and in the compression phase, and a variety of parameters.
\end{abstract}

Keywords: Time enhanced, self-organizing map, image compression, vector quantization, low bitrate, entropy.

\section{Introduction}

Millions of digital images are generated each day: from the few daily shots of an amateur photographer to the countless files at a newspaper's multimedia archive, and there is a real and pressing need to store these images at the lowest possible cost. Furthermore, the advent of the Internet has set a new requirement: the transmission of digital content through bandwidth-limited channels. A very effective, although partial, solution to these problems is the use of lossless and lossy image compression algorithms. There are many lossy compression methods but when high compression ratios are sought codecs based on vector quantization (VQ) represent an attractive alternative because they easily attain $64: 1$ or higher compression ratios without a

* Supported by CONACYT and CONCYTEA, Mexico. 
noticeable quality loss 1]. For the design process of the codebook Linde, Buzo and Gray's algorithm 2] is the classical method, but in the 1980s Kohonen 3] opened the way for a new generation of design processes with his well-known work on artificial neural networks (4] and 5] for example).

The original self organizing map has, however, an inherent limitation: it was designed for spatial representation and can not model the sequential aspects of the data. It is open to discussion whether modeling the sequentiality of the input stream yields better results for a particular problem, but several researchers have modified the original SOM to model non spatial relations 6] -[8]. Campoy et al.[9] developed a novel self organizing map that models the temporal redundancy of the input data: the time enhanced self organizing map (TESOM). By training both a classical map and a TESOM with ordered vectors of known probability density function in $\mathfrak{R}^{2}$, Campoy et al. showed that the time enhanced map had better performance, expressed as a lower entropy of the incremental position in the template index. Here this novel algorithm will be reviewed and compared to a standard SOM for image compression. The capacity of the time enhanced map for lower entropy and equivalent image quality will be showed, as will its ability to learn long and complex sequences.

\section{Time Enhanced SOM Algorithm for VQ Image Compression}

A k-dimensional memoryless vector quantizer (VQ) consists, in general, of a coderdecoder pair: the coder maps input vectors to channel symbols and the decoder maps these symbols to values of a reproduction alphabet. In this particular scenario a finite index set $\mathrm{I}$ of $\mathrm{N}$ consecutive integers, beginning with 0 , represents the channel symbols. The alphabet is a set of carefully selected reproduction vectors, the codebook, which sets the partition of the $\mathfrak{R}^{k}$ space into Voronoi cells through a closest neighbor rule, using the Euclidean distance as the criterion. These conditions define a codec that is fully described by the indexed vectors of the codebook: the compressor matches $w \times h$ subimages to indexed templates and stores the increment of the index, then the decompressor constructs a mosaic with said templates. Therefore the codebook design is critical.

The self organizing map can be used to design VQ codebook, but instead of fully describing the standard algorithm and its modification we will present only the latter and state that for the SOM $R A_{i}(j)=0$. Campoy et al [9] introduced the concept of Residual Activity: each neuron holds energy, which decays with time and is reinforced by the Neighborhood Activity. The Residual Activity changes the criterion for selecting the best matching node: past "winners" are more likely to be selected. The Residual Activity decay is modeled with a Gaussian function and at the beginning of each iteration of the learning algorithm $R A_{i}(j)$ is updated to reflect the energy decay:

$$
R A_{i}(j)=e^{-\frac{\left(1+\sqrt{-2 \sigma_{t}^{2}(j) * \log \left[R A_{i}^{\prime}(j-1)\right]}\right)}{-2 \sigma_{t}^{2}(k)}}
$$

where $R A_{i}^{\prime}$ is the Residual Activity that was reinforced by the Neighborhood Activity at the end of the last iteration: 


$$
R A_{i}^{\prime}(j)=1-\left(1-N A_{i}(j)\right) *\left(1-R A_{i}(j)\right)
$$

This novel modification is remarkable because it does not store several past states of the network; for a full description of the time enhanced map and its properties consult [9].

\subsection{Algorithm}

1. Create the network of $\mathrm{N}$ nodes and set the synaptic weights to random values.

2. Map the input vector $x(j)$ and the synaptic weights $w_{i}(j)$ to $\hat{x}(j)$ and $\widehat{w}_{i}(j)$ in the $\Re^{k+1}$ space:

$$
\hat{v}=\left[v, \widehat{v}_{k+1}\right], \text { where } \hat{v}_{k+1}=\sqrt{1-v_{1}^{2}-v_{2}^{2}-\cdots-v_{k}^{2}}
$$

3. Calculate both the Instantaneous Activity $I A_{i}$ and the Residual Activity $R A_{i}$ for each neuron. Then compute the Total Activity $T A_{i}$ and select the winning node $i_{j}$ :

$$
\begin{gathered}
i_{i}(j)=\max \left(T A_{i}(j)\right), \text { where } T A_{i}(j)=1-\left(1-I A_{i}(j)\right) *\left(1-R A_{i}(j)\right) \text { and } \\
\qquad I A_{i}=\widehat{w}_{i}(j) \bullet \hat{x}_{i}(j)
\end{gathered}
$$

4. Compute the Neighborhood Activity and Learning Factor:

$$
\begin{gathered}
d_{i m}(j)=\left\|P_{i(j)}-P_{m}\right\| \quad \sigma_{v}(j)=\sigma_{v 0} \cdot e^{-j / t_{c}} \quad N A_{i}(j)=e^{-d_{i m}^{2}(j) / 2 \sigma_{v}^{2}(j)} \\
\alpha(j)=\alpha_{0}(j) \cdot e^{-j / t_{c}}
\end{gathered}
$$

where $P_{i(j)}$ is the of the i-th neuron and $P_{m}$ is the index of the winning neuron, both expressed as the position in the output map.

5. Update the Residual Activity $R A_{i}^{\prime}$ (see previous section).

6. Update the synaptic weights:

$$
w_{i}(j+1)=w_{i}(j)+\alpha(j) \cdot N A_{i}(j) \cdot\left[x(j)-w_{i}(j)\right]
$$

7. Iterate from step 2 until $j=5 t_{c}$. No significant adaptation occurs beyond this value.

\section{Benchmarking}

\subsection{Input Data}

For image compression systems, where the probability density function of the data is unknown, it is common to use a large set of vectors from a reference image or group of images. The training vectors are used in no particular order because the SOM only models the similarity of the inputs, but the TESOM needs ordered training data so it can learn its patterns. Obviously the input sequence used for training must be used for image coding, and because there is no previous knowledge on this topic one of the simplest sequences is used, as shown in Fig. 1. As the order of the training vectors is not relevant for the SOM performance the same training data will be used for both design processes. 


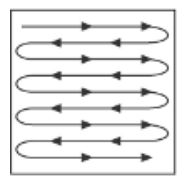

Fig. 1. Input sequence

\subsection{Input Data Correlation}

The VQ compressor transforms a digital image into a set of increments of indexes $A^{\prime}=\left\{i_{0}^{\prime}, i_{1}^{\prime}, i_{2}^{\prime}, \ldots\right\}$. As the input vectors are neighboring partitions of the digital image they are, presumably, correlated: they are similar and there exist sequence patterns. As the TESOM models both the spatial and temporal aspect of the input data vectors close in the input space map to indexes close in the output space, like the typical $\mathrm{SOM}$, but it is also true that vectors close in the input sequence map to a sequence of indexes close in the output space. These rules, while not mutually exclusive, compete at the learning stage and produce a better codebook when the patterns are also present when compressing data.

\subsection{Training parameters}

To train a self organizing map five parameters must be set: the order of the input space, the number of neurons, the characteristic time, the initial neighborhood variance and the initial learning factor. For the TESOM an extra parameter exists: the initial temporal variance. For each parameter a fixed value or range is selected.

The order of the input space is set by the size of the partitions of the digital image: $k=w \times h$, and in this case only the results for $8 \times 8$ and $12 \times 12$ subimages will be reported. Previous experience with SOMs lead to fixed values of the initial neighborhood variance $\left(\sigma_{0}=0.25\right)$ and the initial learning factor $\left(\alpha_{0}=1.5\right)$, as they guarantee a good differentiation and specialization of neurons. The remaining parameters are discussed below.

- $\quad$ Number of neurons $(N)$ : small values boost compression ratio but maximize the quantization error. Since subimage size has a bigger impact in image quality than the value of $N$ standard values may be used: 64, 128 and 256.

- Characteristic time $\left(t_{c}\right)$ : it sets the number of learning iterations $\left(5^{*} t_{c}\right)$. It is assumed that the training data is a large collection of patterns that must be recycled several times so the map will be able to learn them, hence the training data will be recycled 25, 50 and 100 times, being the characteristic time a multiple of the quantity of vectors.

- Initial temporal variance $\left(\sigma_{t 0}\right)$ : in the range $(0, \infty)$ this is the extra parameter of the time enhanced algorithm. For small values the TESOM is less enhanced as the effect of past inputs decays more rapidly, but large values distort the learning process with neurons that win all the time. To gather additional data on the properties of the TESOM 20 different values will be tested: $1-15,20,25,30,35$ and 40 . This means that for each SOM codebook there will be 20 TESOM codebooks with equivalent parameters. 


\subsection{Performance Evaluation}

Two indexes are used to establish the comparison between the algorithms: entropy of the compressed data and the PSNR of the decoded image. The entropy $H\left(A^{\prime}\right)$ is calculated for each compressed image, and for the TESOM-compressed images is reported as a percentage gain or loss against a reference SOM-compressed image:

$$
\Delta=100 *\left(1-H\left(A^{\prime}\right)_{\text {tesom }} / H\left(A^{\prime}\right)_{\text {som }}\right) \% \quad \text {, where } H\left(A^{\prime}\right)=\sum_{j} P\left(i_{j}^{\prime}\right) * \log _{2} P\left(i_{j}^{\prime}\right)
$$

A positive $\Delta$ represents a higher compression ratio of the TESOM algorithm. This is possible because the time enhanced algorithm has an extra training parameter $\left(\sigma_{t 0}\right)$ with 20 different values, so each one of those maps may be compared to one SOM with identical standard parameters.

\section{Results}

The full suite of tests comprises 4,326 codebooks: 7 images were used for training and 13 images for compression test over a wide range of training/coding parameters, that has yield to 60,564 different experiments for compression of 8bpp (grayscale) images. A subset of the compressed images is shown in Fig. 2. From left to right: Lena $(512 \times 512$ pixels), Mandrill $(512 \times 512)$, self-portrait $(400 \times 500)$ of Dutch neoimpressionist Vincent van Gogh, "Woman with parasol" $(288 \times 360)$ and "Woman with parasol - Madame Monet and her son" $(268 \times 400)$ of French impressionist Claude Monet. They will be referred hereafter as lena, mand, gogh, monl and mon2, respectively. It is important to note that the training data is a set of subimages (quarter of total area) of the test images.

The presented results are clustered into three possible scenarios: best-case, where the compressed image fully contains the training image; normal-case, where the compressed image is of the same class (i.e. visually similar) as the training image (i.e. gogh and monl); and a worst-case, where the compressed image is not related to the training image.
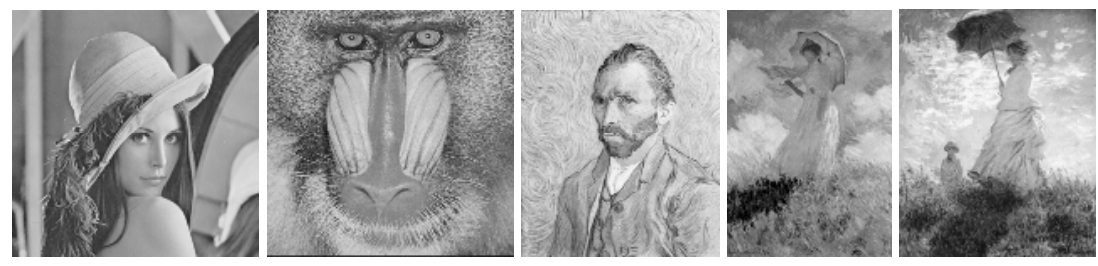

Fig. 2. Subset of test images

The first battery of tests uses $12 \times 12$ subimages and reveals that the TESOM algorithm has an average of $10 \%$ performance advantage over the SOM in terms of the Entropy of the code increment, i.e. in actual terms of bitrate. The leftmost graph of 
Fig. 3 shows the better compression of the TESOM for a range of $\sigma_{t 0}$ values when a lena-subimage is used to train the map and lena and mand are compressed. The image quality is equivalent for both cases, as shown as values of PSNR in Table 1 and visually in Fig. 4. The middle graph of Fig. 3 shows $a$ similar situation when training with a mand-subimage, and the results are consistent with the previous test. These graphs demonstrate both the best and worst case scenarios with two unrelated images while the rightmost graph of Fig. 3 presents the results for a normal scenario where one image is representative of the group of images to be compressed and shows even better performance (see Table 1 for the image quality comparison).

Compression with smaller templates of $8 \times 8$ pixels leads to a smaller TESOM advantage, down to an average value of $6 \%$ in the bitrate. Image quality is still equivalent, and even if in some cases the TESOM performs equally than a SOM it can be said that in general the time enhanced algorithm keeps a small lead in performance.
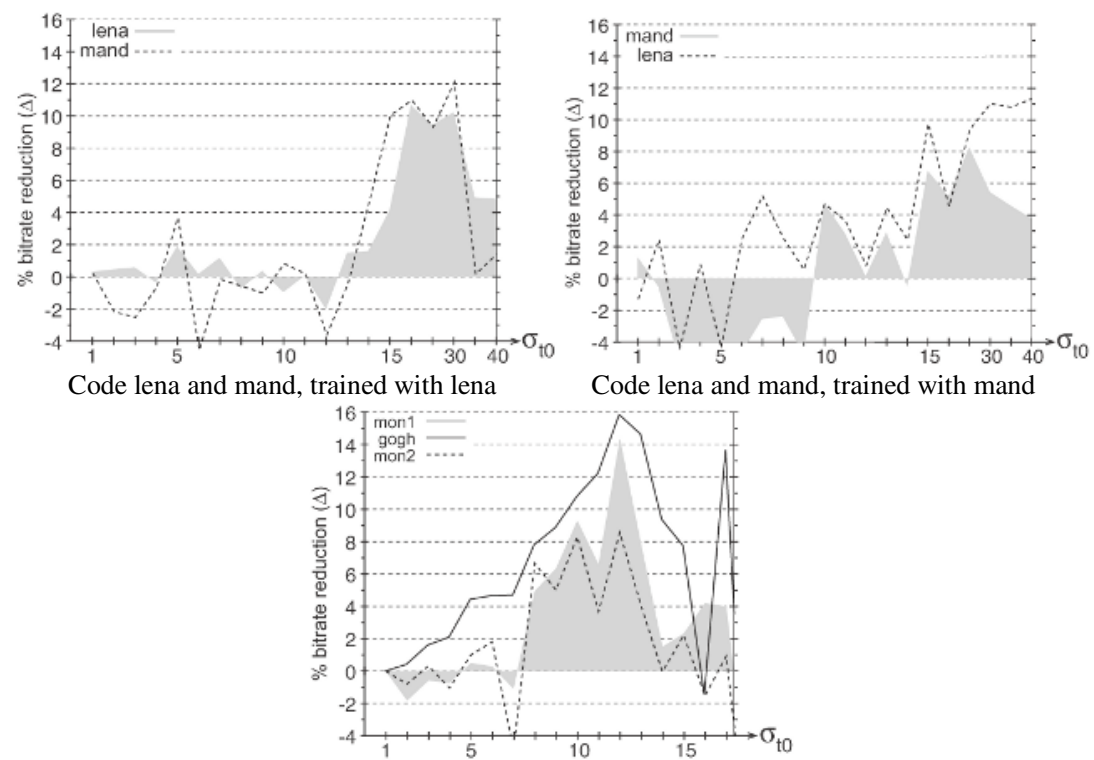

Code mon1-2 and gogh, trained with mon1

Fig. 3. Bitrate reduction for $12 \times 12$ subimages and $N=128$

Table 1. Image quality comparison in $\mathrm{dB}$ (PSNR)

\begin{tabular}{|cccc|}
\hline $\begin{array}{c}\text { Training } \\
\text { subimage }\end{array}$ & Compressed image & $\begin{array}{c}\text { SOM-coded } \\
\text { PSNR }\end{array}$ & $\begin{array}{c}\text { TESOM-coded } \\
\text { average PSNR }\end{array}$ \\
\hline lena & Lena & 23.55 & 23.46 \\
12x12 & mand & 18.42 & 18.42 \\
\hline mand & mand & 18.62 & 18.61 \\
12x12 & lena & 21.62 & 21.77 \\
\hline monl & monl & 22.10 & 22.08 \\
$12 \times 12$ & gogh & 21.14 & 21.05 \\
& mon2 & 21.56 & 21.53 \\
\hline \hline
\end{tabular}




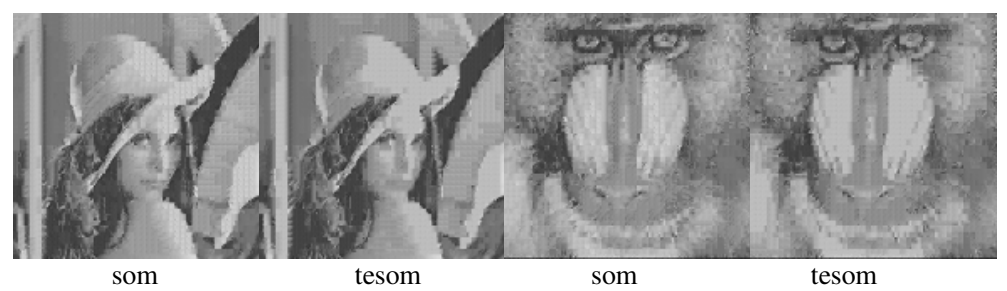

Fig. 4. Visual results: TESOM vs. SOM (trained with lena, $12 \times 12$ subimages, $\sigma_{t 0}=30$ )

\section{Conclusions}

A new and innovative modification to Kohonen's algorithm, the time enhanced self organizing map TESOM, has been reviewed and its application extended for the design of VQ codebooks for digital image compression. The performance of the time enhanced algorithm was compared to that of a standard SOM using two quality indexes: the compression ratio (measured as the entropy of the of the code increment) and the objective quality of the decoded images (PSNR).

Tests show that maps obtained by TESOM are able to learn the sequence order of th long and complex sequences that exist in the training data (i.e. subimages). This additional information is taken into account for a better ordering of the vectors in the codebook, which leads to a performance advantage when coding data with similar patterns. This bitrate improvement is most remarkable when compressing with big subimages $(12 \times 12$ pixels), when the subimages are not very similar and the sequential order is therefore more relevant.

It is important to note that the TESOM algorithm introduces only one extra training parameter and that its value is not critical, since a good performance is possible for a wide range of temporal variances $10 \leq \sigma_{t 0} \leq 30$. Furthermore, typically the worst performing TESOM is equivalent to the reference SOM in both compression ratio and image quality. Additionally better performance was demonstrated for the three scenarios tested: a best case where the compressed image fully contains the training data, a normal case where the compressed image is of the same class as the training data and a worst case where the compressed image is not related to the training data.

Future work will focus on different ordering sequences of the subimages, video compression and enhancements to the algorithm based on the knowledge and data acquired with the experiments and results.

\section{References}

1. N. M. Nasrabadi, R. A. King, Image coding using vector quantization: a review, IEEE Transactions on Communications 36 (8) (1988) 957-971

2. Y. Linde, A. Buzo, R. M. Gray, An algorithm for vector quantizer design, IEEE Transactions on Communications 28 (1) (1980) 84-95

3. T. Kohonen, The self-organizing map, Proceedings of the IEEE 78 (9) (1990) 1464-1480

4. H.-C. Wei, Y.-C. Chang, J.-S. Wang, A Kohonen-based structured codebook design for image compression, in: TENCON '93 IEEE Region 10 Conference on Computer Communication, Control and Power Engineering, Proceedings of, Vol. 3, 1993, pp. 426-429 
5. C. Amerijckx, M. Verleysen, P. Thissen, J.-D. Legat, Image compression by selforganized kohonen map, IEEE Transactions on Neural Networks 9 (3) (2002) 503-507

6. Kangas, Phoneme recognition using time-dependent versions of selforganizing maps, in: IEEE International Conference on Acoustics, Speech and Signal Processing, Proceedings of, 1991, pp. 101-104

7. L. James, R. Miikkulainen, SARDNET: A self-organizing feature map for sequences, in: NIPS'94 Advances in Neural Information Processing Systems, Proceedings of, 1994, pp. 577-584

8. R. Euliano, J. C. Principe, Spatiotemporal self-organizing feature maps, in: International Joint Conference on Neural Networks, Proceedings of, Vol. 4, 1996, pp. 1900-1905

9. Campoy, C. J. Vicente, Residual Activity in neurons allows SOMs to learn temporal order, International Conference on Artificial Neural Networks, September 11-15, 2005, Warsaw, Poland. 